\title{
COMMENT
}

\section{A multilevel-based research framework on congenital Zika syndrome}

\author{
Marcio Leyser $\mathbb{D}^{1}{ }^{1}$, Fernanda J. P. Marques ${ }^{2}$ and Osvaldo J. M. Nascimento ${ }^{2}$ \\ Pediatric Research (2019) 85:745-746; https://doi.org/10.1038/s41390-019-0349-0
}

In 2015, the spotlights were directed towards Brazil when the country experienced an unprecedented epidemic of Zika virus (ZIKV) infection. ${ }^{1}$ Since then, much has been studied on the pathophysiological mechanisms by which ZIKV is transmitted, gains access to the nervous system, replicates in the human host, and damages neuronal cells and their precursors. ${ }^{2}$

Additionally, a growing body of scientific articles has demonstrated the clinical presentations of infants affected by congenital Zika syndrome (CZS). Severe motor delays, functional impairments, and a strong risk factor to the comorbid diagnosis of cerebral palsy are some of the features described in recent studies. ${ }^{3}$ It has been postulated that infants who do not show any clinical manifestations at birth might display neurodevelopmental abnormalities, including learning and memory deficits, in their late childhood years. ${ }^{4}$

As the likelihood of a second wave of ZIKV epidemic remains high, designing predictive models to assess CZS severity is urgently necessary. Moreover, as children affected by CZS are growing, some of whom are now 3 years of age, it is time to invest in studies that will look further into how health care professionals can optimize an adequate array of resources that would define directives for goaloriented early stimulation programs in this population according to their clinical presentations and psychosocial needs.

We propose the creation of a multilevel-based research framework on CZS that could possibly result in predictive models that would be likely beneficial for the early identification of newborns and infants born with CZS who are at higher risk for disease burden, including, for example, the risk of cerebral palsy and autism spectrum disorders.

These studies should be organized based on the multifaceted medical aspects that, together, form the still-enigmatic CZS's biopsychosocial gestalt. Ideally, the research comprising the framework should follow a bottom-up timeline progression according to the unfolding new clinical observations found in Zika cohorts over time as the studies' participants grow and develop. Some examples of what has been already accomplished and future perspectives on CZS research, according to each level of the framework, are described below and illustrated in Fig. 1.

\section{LEVEL 1: EPIDEMIOLOGICAL}

The association between regional meteorological changes and the proliferation of the Aedes sp.; the association between the Aedes aegypti mosquitoes and CZS; the association between pregnancy and laboratory-confirmed ZIKV infection. ${ }^{1}$

\section{LEVEL 2: CLINICAL}

Patterns of congenital anomalies secondary to central and peripheral nervous system involvement. The distinctive phenotype of CZS including severe microcephaly with partially collapsed skull, thin cerebral cortices with subcortical calcifications, macular scarring and focal pigmentary mottling, congenital contractures, marked early hypertonia, and symptoms of extrapyramidal involvement.

\section{LEVEL 3: NEUROIMAGING}

Due to limited laboratory-related technical diagnostic accuracy of ZIKV exposure in asymptomatic pregnant women, as well as the lack of specific serology tests for ZIKV, the unique radiological pattern observed in infants with CZS can play an important role in the early diagnosis of this condition.

Pre-natal ultrasonography (US) screening findings: brain calcifications, cerebral atrophy, posterior fossa and corpus callosum abnormalities, ventriculomegaly. In addition, measurement of the head circumference is a crucial evaluation and one of the main clinical evaluations to detect microcephaly.

Post-natal US findings: thinning of the frontoparietal parenchyma with punctiform hyperechoic findings consistent with calcifications and mainly observed on the frontal brain surfaces, dysgenesis of the corpus callosum.

Computed tomography findings: microcephaly, exuberant external occipital protuberance, reduced frontonasal angle and redundant scalp skin, premature closure of cranial sutures, brain calcifications specifically in the junction between the cortex and the subcortical white matter.

Magnetic resonance imaging findings: malformations of cortical development and sulcation, simplified pattern of cortical sulci and gyri and normal cortical thickness, associated with areas of polymicrogyria and/or pachygyria with a thick cortex predominantly observed in the frontal lobes. ${ }^{5}$

\section{LEVEL 4: LABORATORY}

The laboratory criteria for the confirmation of ZIKV infection is based on the detection of the virus in the blood (acute phase) and urine (after the first week of symptoms). Real-time reverse transcription-polymerase chain reaction analysis contributes to detect the viral RNA. The diagnosis might also be assessed with

\footnotetext{
${ }^{1}$ Department of Pediatrics, University of lowa, lowa City, IA, USA and ${ }^{2}$ Department of Neurology, Antonio Pedro University Hospital/Fluminense Federal University, Niterói, Rio de Janeiro, Brazil

Correspondence: Marcio Leyser (marcio-leyser@uiowa.edu)
}

Received: 23 January 2019 Accepted: 13 February 2019

Published online: 21 February 2019 


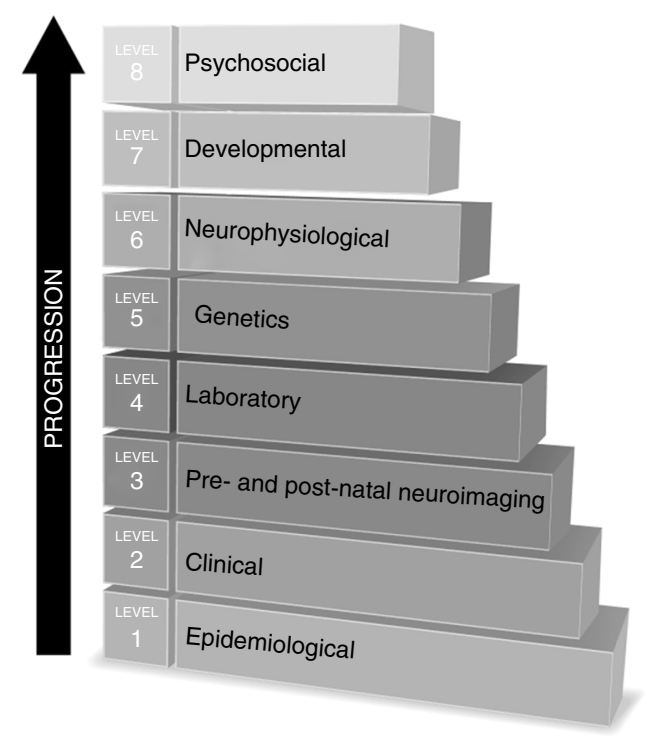

Fig. 1 Timeline of what has been already accomplished and future perspectives on CZS research based on a multilevel system for the creation of predictive models of severity of CZS

analysis for immunoglobulin $M(\lg M)$ and the plaque reduction neutralization test for antibodies against the virus. In some cases, the diagnosis is established retrospectively and confirmed by serologic assays. ${ }^{5}$

\section{LEVEL 5: GENETICS}

The identification of protective genes might play an important role in the pathophysiology of ZIKV infection. A recent study using CRISPR technology ${ }^{6}$ identified the IFI6 gene as a potent antiviral gene targeting flaviviruses. The researchers used traditional cell culture studies to confirm the gene's role in protecting against infection by Zika, West Nile, dengue, and yellow fever viruses.

\section{LEVEL 6: NEUROPHYSIOLOGY}

Due to the cell damage caused by ZIKV in the central nervous system, ${ }^{2}$ seizures can be a clinical manifestation in the early ages. The diagnosis of epilepsy in infants with CZS can be linked to a poor prognosis in their neurodevelopmental outcomes.

\section{LEVEL 7: DEVELOPMENTAL}

The impact on motor, language, cognition, social-communication skills, and behavior, ${ }^{4}$ in addition to a higher risk for the frequently found comorbid diagnosis of cerebral palsy, ${ }^{3}$ might be early determined by interdisciplinary evaluation. Individualized, goaloriented, and family-centered rehabilitation programs could be designed based on these measurements.

\section{LEVEL 8: PSYCHOSOCIAL}

The severity of the abnormalities at birth-coupled with the anticipation of a lifetime of caregiving and economic burdens, uncertainty about the unfolding developmental and medical consequences, social isolation, and potential stigma-can lead to high levels of family distress. The support to caregivers requires a family-centered approach, extensive care coordination, access to evolving new information, ongoing surveillance, and individualized planning for child and family services. ${ }^{7}$

As knowledge on ZIKV progresses, data generated through this proposed framework might be used to create binary predictive modules that ultimately will contribute to the diagnostic accuracy of CZS, the neurodevelopmental prognostication of infants born with this novel congenital infectious disease, and the determination of those who would benefit from goal-oriented early interventions.

\section{ACKNOWLEDGEMENTS}

We thank Mr. Paul J Casella for his help with the editing of this manuscript and Mrs. Leigh Bradford for her help with the designing of the figure.

\section{AUTHOR CONTRIBUTION}

M.L. conceptualized, designed the article, drafted the initial manuscript, and reviewed and revised the manuscript critically for important intellectual content. F.J.P.M. drafted the initial manuscript, and reviewed and revised the manuscript critically for important intellectual content. O.J.M.N. made substantial contributions to the conception of this article, reviewed and revised the manuscript critically for important intellectual content, and gave final approval of the version to be published.

\section{ADDITIONAL INFORMATION}

Competing interests: The authors declare no competing interests.

Publisher's note: Springer Nature remains neutral with regard to jurisdictional claims in published maps and institutional affiliations.

\section{REFERENCES}

1. da Silva Pone, M. V. et al. Zika virus infection in children: epidemiology and clinical manifestations. Child's Nerv. Syst. 34, 63-71 (2018).

2. Chimelli, L. et al. The spectrum of neuropathological changes associated with congenital Zika virus infection. Acta Neuropathol. 133, 983-999 (2017).

3. Marques, F. J., et al. Children Born with Congenital Zika Syndrome Display Atypical Gross Motor Development and a Higher Risk for Cerebral Palsy. J. Child Neurology. 2018; https://doi.org/10.1177/0883073818811234

4. Moor Wheeler, A. C. Development of infants with congenital Zika syndrome: what do we know and what can we expect? Pediatrics 141 (Supplement 2), S154-S160 (2018).

5. Ribeiro, B. G. et al. Central nervous system effects of intrauterine Zika virus infection: a pictorial review. Radiographics 37, 1840-1850 (2017).

6. Richardson, R. B. et al. A CRISPR screen identifies IFI6 as an ER-resident interferon effector that blocks flavivirus replication. Nat. Microbiol. 3, 1214 (2018).

7. Bailey, D. B. \& Ventura, L. O. The likely impact of congenital Zika yndrome on families: considerations for family supports and services. Pediatrics $\mathbf{1 4 1}$ (Supplement 2), S180-S187 (2018). 